\title{
Correction to: Changes in relative fit of human heat stress indices to cardiovascular, respiratory, and renal hospitalizations across five Australian urban populations
}

\author{
James Goldie ${ }^{1,2,3} \cdot$ Lisa Alexander ${ }^{1,2} \cdot$ Sophie C. Lewis ${ }^{2,3} \cdot$ Steven C. Sherwood ${ }^{1,2} \cdot$ Hilary Bambrick $^{4}$
}

Published online: 1 March 2019

(C) ISB 2019

\section{Correction to: International Journal of Biometeorology: (2018) 62: 423 https://doi.org/10.1007/s00484-017-1451-9}

The authors of the article would like to bring the following correction/corrigendum to attention:

When recently investigating future changes in heat stress indices, we discovered an error in the use of the heatwave indices we compared in Goldie et al. (2017). The heatwave index formulae listed in Table 1 of Goldie et al. (2017) are correct; rather, we incorrectly lagged them for use in our analysis. We stated in the article that we chose to lag the heatwave indices by 2 days because they are designed for forecasting use and hence use forecast data from the day of exposure to 2 days later. In the context of our analysis, they would instead use data from 2 days prior to the day of exposure.

However, lagging the indices after calculating them does not correctly realise this intent: temperature information from 2 days after an exposure is still being compared to the response (hospital admission). Instead, the input temperature series should have been lagged before we used them to calculate the heatwave indices. Although correcting this error has not altered the main conclusions

The online version of the original article can be found at https://doi.org/ 10.1007/s00484-017-1451-9

James Goldie

j.goldie@unsw.edu.au

1 Climate Change Research Centre, UNSW Australia, Sydney, NSW, Australia

2 ARC Centre of Excellence for Climate System Science, UNSW Australia, Sydney, NSW, Australia

3 Fenner School of Environment \& Society, Australian National University, Acton, ACT, Australia

4 School of Public Health and Social Work, Queensland University of Technology, Brisbane, QLD, Australia of our analysis, some of the details of our results did change. The ranks of heatwave indices compared to other indices (Table 2 corrected), as well as the size of their effects of on modelled hospital admissions (Table 3 corrected), have shifted in most cases. However, none of the effects have changed sign, bar one: the effect of 3-Day Maximum Temperature (3DMT) on renal admissions in Sydney, which was not, and still is not, statistically significant.

Updating these results did also cause some changes to the statistical significance of heatwave indices. Most importantly, two of the heat stress indices-3-Day Average Temperature (3DAT) and 3DMT - are statistically significant against cardiovascular admissions in Sydney, where none were before, and no heat stress indices are statistically significant against cardiovascular admissions in Cairns, where one - 3DMT - was before. We noted the difficulty of modelling cardiovascular admissions in the original analysis; these changes suggest that the effects of heat stress indices in these cases may be less robust.

Correcting this error has not altered the main conclusions of our analysis: in fact, we believe these updated reports more strongly support the conclusion that the best heat stress indices in Australian cities vary primarily according to the disease group modelled.

The original article has been corrected.

\section{Reference}

Goldie J, Alexander L, Lewis SC et al (2017) Changes in relative fit of human heat stress indices to cardiovascular, respiratory, and renal hospitalizations across five Australian urban populations. Int J Biometeorol:1-10. https://doi.org/10.1007/s00484-017-1451-9 